\title{
Cuantificación de Cobre en Polifenoloxidasa de Frutas Tropicales por Espectrofotometría de Absorción Atómica
}

\author{
María E. Jiménez-Vieyra ${ }^{(1)}$ y María L. Zambrano-Zaragoza ${ }^{(2)}$ \\ (1) Escuela Superior de Ingeniería Química e Industrias Extractivas, Instituto Politécnico Nacional. \\ Unidad Profesional Adolfo López Mateos, Edificio 7, Zacatenco, 07738, México \\ (e-mail: mejimenezv@gmail.com) \\ (2) Facultad de Estudios Superiores, Cuautitlán, Universidad Nacional Autónoma de México, \\ Av. Primero de mayo s/n, Col. Atlanta, Cuautitlán Izcalli, 54740, México \\ (e-mail: luz.zambrano@unam.mx)
}

Recibido May. 11, 2010; Aceptado Jul. 12, 2010; Versión Final recibida Ago. 31, 2010

\begin{abstract}
Resumen
Este estudio cuantifica el contenido de cobre en aguacate y su pH en ciruela, fresa, kiwi, mamey, mango, manzana, pera y plátano. También cobre en la enzima polifenoloxidasa y velocidad de obscurecimiento. El cobre se determinó mediante espectrofotometría de absorción atómica en muestras digeridas por vía húmeda. El aguacate tuvo más cobre en enzimas $(0.059 \mathrm{mg})$ con mayor velocidad de obscurecimiento $\left(0.751 \mathrm{~s}^{-1}\right)$; la fresa tuvo la menor cantidad de cobre (0.016 $\mathrm{mg}$ ) y la menor velocidad de reacción $\left(0.115 \mathrm{~s}^{-1}\right)$. Las frutas con $\mathrm{pH}$ más ácido tuvieron contenido medio de cobre en la enzima y el menor obscurecimiento. La pera y el plátano tuvieron velocidad de reacción (0.3) y pH similares pero contenidos diferentes de cobre. Los resultados permiten establecer una correlación directa entre el contenido de cobre en enzimas y la velocidad obscurecimiento.
\end{abstract}

Palabras clave: absorción atómica, cobre, frutas, polifenoloxidasa, velocidad de obscurecimiento

\section{Copper Quantification on Tropical Fruits by Atomic Absorption Spectrophotometry}

\begin{abstract}
This work quantifies the copper content in avocado and $\mathrm{pH}$ in, plum, strawberry, kiwi, mamey sapote, mango, apple, pear and banana. Copper and browning rate on enzymes of those fruits were also determined. Copper content was measured by atomic absorption spectrophotometry on samples digested by wet-chemical method. Avocados had the highest content of copper on enzymes $(0.059 \mathrm{mg})$ with the highest browning rate $\left(0.751 \mathrm{~s}^{-1}\right)$ while strawberries had the lowest copper content $(0.016 \mathrm{mg})$ and the lowest browning rate $\left(0.115 \mathrm{~s}^{-1}\right)$. Fruits with acid $\mathrm{pH}$ had acceptable copper content on the enzyme and a low browning rate. Pears and bananas had similar $\mathrm{pH}$ and browning rate (0.3) with different copper contents. The results do not allow establishing a direct relationship between copper content in enzymes and its browning rate.
\end{abstract}

Keywords: atomic absorption, copper, fruit, polyphenoloxidase, browning rate 


\section{INTRODUCCIÓN}

La actividad fisiológica de frutas y verduras es el resultado de varios factores como la especie y variedad, la edad de los frutos, el $\mathrm{pH}$, la temperatura, la actividad de las enzimas oxidativas, la disponibilidad de $\mathrm{O}_{2}$, la naturaleza y cantidad de los substratos fenólicos y, los cofactores como los iones metálicos $\mathrm{Fe}^{3+}$ y $\mathrm{Cu}^{2+}$ (Artés et al., 1998). En términos de enzimas oxidativas, la Polifenoloxidasa (PFO) es una enzima de interés ya que influye en diversas respuestas biológicas. Esta enzima y los polifenoles en los alimentos reaccionan en presencia de oxígeno molecular obscureciendo visiblemente el producto (Queiroz et al., 2008).

Las reacciones oxidativas pueden inhibirse o precipitarse en presencia de cofactores. Diversas investigaciones han identificado al cobre como un cofactor que incrementa la actividad de la PFO. En las uvas se ha identificado una correlación entre el obscurecimiento de soluciones hidroalcohólicas de catequín y la concentración de iones cúpricos (Es-Safi et al., 2003).

Frecuentemente, los metales pesados están presentes en los alimentos como trazas o ultratrazas. Hay técnicas analíticas con suficiente sensibilidad para la determinación exacta de estos elementos químicos en muestras de alimentos. Las técnicas empleadas con mayor frecuencia son la espectrofotometría de absorción atómica por flama, por horno de grafito o por vapor frío y la espectrofotometría de emisión por plasma inductivo o por plasma inductivo acoplado a masas. La espectrofotometría de emisión por plasma tiene un elevado rango de detección (Sahan et al., 2007) pero resulta muy cara si se desea determinar uno o dos elementos.

Existen otras técnicas para la detección de metales. El análisis por activación del neutrón permite cuantificar simultáneamente muchos elementos en muestras de pocos miligramos o de varios kilos (Zukowska y Biziuk, 2008), pero es una técnica menos utilizada porque es necesario tener acceso a un reactor para producir los neutrones.

La selección de la técnica de detección de metales depende de varios factores, como la rapidez deseada, la exactitud y sensibilidad del análisis, la facilidad de uso y el costo. La espectrofotometría de plasma inductivo acoplado a masas ofrece ventajas sobre la de absorción atómica ya que ofrece un análisis simultáneo de multielementos con un alto rango de detección; pero en la determinación de tan sólo un par de elementos, la segunda técnica tiene menor costo.

En los métodos espectrofotométricos, las muestras de alimentos deben prepararse en solución. La digestión es un método de pretratamiento de la muestra utilizado para destruir la matriz orgánica y conservarla en solución. El método convencional por vía húmeda incluye ácido nítrico o sulfúrico y utiliza un digestor Kjeldahl con plato de calentamiento; la vía seca requiere la formación de cenizas y la recuperación de metales con un ácido. Una tercera alternativa es llevar a cabo la digestión asistida con microondas, con la que el tiempo de digestión se reduce (Zukowska y Biziuk, 2008; Pedro et al., 2006).

Este trabajo cuantificó el contenido de cobre y el pH en muestras de aguacate, ciruela, fresa, kiwi, mamey, mango, manzana, pera y plátano. Además se cuantificó el contenido de ese metal en el extracto de enzimas de las muestras y se determinó la actividad de la enzima PFO.

\section{MATERIALES Y MÉTODOS}

Las frutas utilizadas para este estudio fueron seleccionadas en función de la sensibilidad al ambiente, composición y características. Las frutas utilizadas fueron aguacate (Persea americana), ciruela (Spondias spp), fresa (Fragaria spp), kiwi (Actinidia chinesis), mamey (Colocarpum mammosum), mango (Mangifera indica), manzana (Malus sylvestris Mill), pera (Pirus comunis L.) y plátano (Musa sapientum).

El método seleccionado para la detección de contenido en partes por millón de cobre en las frutas y sus enzimas fue la espectrofotometría de absorción atómica por llama. Este método resulta accesible para la detección de uno o dos elementos químicos cuando se cuenta con amplia 
disponibilidad de las muestras. Es decir, este método utiliza mayores cantidades de muestra para detectar metales con sensibilidad de partes por millón, el metal de la muestra para ser analizado debe de estar en solución. En contraste, la espectrofotometría de emisión por plasma inductivo acoplado a masas tiene una sensibilidad en partes por billón que requiere cantidades mínimas de la muestra pero el equipo y su uso tienen un alto costo.

El espectrofotómetro de absorción atómica (AAnalyst100, Perkín Elmer) se calibró con una solución de 4 ppm de cobre preparada por dilución a partir de una solución madre de 1000 ppm de calidad certificada en contenido de cobre (Sigma Aldrich, Croftsman in Chemistry Milwawkee Wis 532333 USA, catálogo 20,723-3). La solución de 4 ppm de cobre produce una señal de absorbancia de $0.250 \pm 20 \%$. Se crean disoluciones subsecuentes de cobre para obtener concentraciones conocidas, de 0.05 ppm a 0.40 ppm. Estas disoluciones se utilizan para construir la curva de calibración con las absorbancias en el eje horizontal y las concentraciones de los estándares en ppm para el eje vertical. Esta calibración permite estimar la cantidad de cobre en ppm de las muestras de fruta digeridas y aforadas a $100 \mathrm{~mL}$. El rango de detección del cobre en este espectrofotómetro va de 0.67×10-3 ppm a 5 ppm (Citak, Tuzen, 2010; Onianwa, et al, 1999; Sapp y Davidson, 1991).

La Norma Oficial Mexicana NOM-117SSA1-1994 para la determinación de metales en alimentos fue la base teórica del proceso. Para la cuantificación de cobre, se prepararon muestras por triplicado de las frutas mencionadas. Las muestras sufrieron un pretratamiento de oxidación por vía húmeda: a las muestras de $10 \mathrm{~g}$ de fruta se añadieron $10 \mathrm{~mL}$ de ácido nítrico concentrado (Latís Chemical) con peso específico de 1.42 y pureza de 69 \%. Las muestras en solución se colocaron en un digestor Kjeldhal de vidrio Pyrex abierto convencional con placa de calentamiento bajo una campana extractora. La digestión se llevó a cabo por 3 horas a $75{ }^{\circ} \mathrm{C}$ hasta que la solución se tornó transparente. La solución se filtró y aforó a $100 \mathrm{~mL}$ con agua desionizada. Se realiza un blanco que siguió el mismo proceso.

La nueva solución aforada se introdujo al espectrofotómetro que incluye una lámpara de cátodo hueco de cobre que opera a una longitud de onda de $324.8 \mathrm{~nm}$, un slit de $0.7 \mathrm{~nm}$ y $10 \mathrm{~cm}$ de base del quemador en donde se llevó a cabo la atomización de las muestras. La mezcla de gases en el equipo usó aire como oxidante y acetileno como combustible. Los resultados del espectrofotómetro se registraron en términos de absorbancia que se traducen a ppm de acuerdo a la curva de calibración.

Para determinar el $\mathrm{pH}$ de las frutas se tomaron muestras de $10 \mathrm{~g}$ y se agregaron $100 \mathrm{~mL}$ de agua desionizada fría. La mezcla se maceró por $30 \mathrm{~min}$, agitando ocasionalmente y decantando el líquido sobrante. La pulpa quedó en reposo por $10 \mathrm{~min}$. El pH se determinó con ayuda de un potenciómetro (Conductronic, modelo $\mathrm{pH}$ 10) calibrado previamente con soluciones buffer a dos puntos: pH 4 y pH 7 (Marca Baker) (AOAC, 1984).

Por otro lado, la obtención de la enzima cruda de Polifenoloxidasa (PFO) requirió nuevas muestras de $60 \mathrm{~g}$ para cada fruta. A cada muestra se agregaron $90 \mathrm{~mL}$ de buffer de fosfato de sodio $\left(\mathrm{Na}_{2} \mathrm{HPO}_{4} / \mathrm{NaH}_{2} \mathrm{PO}_{4}\right) 0.2 \mathrm{M}$ con $\mathrm{PH}$ de 6.5 conteniendo $5 \%(\mathrm{w} / \mathrm{v})$ de polivinilpirrolidina de Sigma Aldrich (Fang et al., 2007; Cano et al., 1990). Esta solución se homogeneizó con $100 \mathrm{~mL}$ de agua desionizada helada y se filtró con gasa de algodón. El líquido resultante fue centrifugado a $20000 \times \mathrm{g}$ por $20 \mathrm{~min}$ a $4^{\circ} \mathrm{C}$ hasta obtener $102 \mathrm{~mL}$ de extracto de enzimas (Zukowska y Biziuk, 2008; Sapp y Davidson, 1991). La obtención de enzimas se realizó por triplicado para cada fruta.

Cien $\mathrm{mL}$ de extracto no purificado fueron digeridos con $10 \mathrm{~mL}$ de ácido nítrico concentrado por método convencional en un digestor Kjeldhal de Pyrex abierto con placa de calentamiento bajo una campana extractora. La digestión se lleva a cabo por 3 horas a $75^{\circ} \mathrm{C}$ hasta que la solución se tornó transparente. La solución se filtró y aforó a $100 \mathrm{~mL}$ con agua desionizada. Se realiza un blanco que siguió el mismo proceso. Esta solución digerida fue introducida al espectrofotómetro para cuantificar el cobre contenido en ella. 
Además de cuantificar la cantidad de cobre en las enzimas extraídas de cada fruta, se determinó la actividad de la PFO por espectrofotometría ultravioleta-visible (Lambda 2, Perkín Elmer). A $2 \mathrm{~mL}$ del extracto no purificado se añaden $1 \mathrm{~mL}$ de buffer de fosfato de sodio $(0.2 \mathrm{M}, \mathrm{pH} 6.5), 1 \mathrm{~mL}$ de solución alcohólica al $1 \%$ de catecol (Sigma Aldrich, pureza de $99 \%$ ) y $22 \mathrm{~mL}$ de agua desionizada. Esta solución se coloca en el espectrofotómetro UV-Visible para obtener las absorbancias a $420 \mathrm{~nm}$. El blanco es idéntico a la solución descrita pero $1 \mathrm{~mL}$ de catecol es reemplazado por $1 \mathrm{~mL}$ de agua desionizada (Rangana, 1986; Lamikanra y Watson, 2001; Fang et al., 2007).

\section{RESULTADOS Y DISCUSIÓN}

La Tabla 1 presenta el pH y los resultados de la cuantificación de cobre en la fruta cruda y en el extracto de enzimas de ésta. La curva de calibración del espectrofotómetro permite obtener resultados en ppm y éstos pueden convertirse a mg de Cu por $100 \mathrm{~g}$ de muestra. De las frutas analizadas, el aguacate alcanzó la mayor concentración de cobre en pulpa con 0.292 mg por cada $100 \mathrm{~g}$ de muestra, y en extracto enzimático con $0.059 \mathrm{mg}$; el pH detectado fue 7.12.

Tabla 1: Cuantificación de cobre en frutas por absorción atómica

\begin{tabular}{|l|c|c|c|c|c|}
\hline \multirow{2}{*}{ Fruta } & \multirow{2}{*}{$\mathrm{pH}$} & \multicolumn{2}{|c|}{ Cu en fruta $(\mathrm{n}=3)$} & \multicolumn{2}{c|}{ Cu en enzima de fruta $(\mathrm{n}=3)$} \\
\cline { 3 - 6 } & & $\mathrm{ppm}$ & $\mathrm{mg} / 100 \mathrm{~g}$ & $\mathrm{ppm}$ & $\mathrm{mg} / 100 \mathrm{~g}$ \\
\hline Aguacate & $7.12 \pm 0.10$ & $0.292 \pm 0.12$ & $0.292 \pm 0.030$ & $0.355 \pm 0.30$ & $0.059 \pm 0.012$ \\
\hline Ciruela & $4.07 \pm 0.05$ & $0.119 \pm 0.23$ & $0.119 \pm 0.020$ & $0.198 \pm 0.23$ & $0.033 \pm 0.010$ \\
\hline Fresa & $4.03 \pm 0.04$ & $0.094 \pm 0.06$ & $0.094 \pm 0.010$ & $0.099 \pm 0.07$ & $0.016 \pm 0.012$ \\
\hline Kiwi & $3.66 \pm 0.12$ & $0.130 \pm 0.21$ & $0.130 \pm 0.030$ & $0.304 \pm 0.15$ & $0.050 \pm 0.014$ \\
\hline Mamey & $7.25 \pm 0.13$ & $0.038 \pm 0.27$ & $0.038 \pm 0.019$ & $0.083 \pm 0.09$ & $0.013 \pm 0.011$ \\
\hline Mango & $4.89 \pm 0.10$ & $0.191 \pm 0.10$ & $0.191 \pm 0.030$ & $0.258 \pm 0.18$ & $0.043 \pm 0.010$ \\
\hline Manzana & $5.45 \pm 0.20$ & $0.072 \pm 0.05$ & $0.072 \pm 0.020$ & $0.190 \pm 0.28$ & $0.031 \pm 0.012$ \\
\hline Pera & $5.54 \pm 0.20$ & $0.186 \pm 0.22$ & $0.186 \pm 0.030$ & $0.310 \pm 0.17$ & $0.051 \pm 0.012$ \\
\hline Plátano & $5.60 \pm 0.30$ & $0.139 \pm 0.35$ & $0.139 \pm 0.010$ & $0.176 \pm 0.29$ & $0.029 \pm 0.013$ \\
\hline
\end{tabular}

El cuerpo humano requiere el consumo de $1.0 \mathrm{mg}$ de cobre al día para alcanzar un efecto protector contra algunas enfermedades (Joo y Betts, 1996); el aguacate apenas contribuye en un $30 \%$ del consumo sugerido. La literatura identifica otros alimentos con alto contenido de cobre que tampoco rebasan el consumo sugerido: investigaciones con bebidas de cocoa con pretratamiento de digestión y cuantificación por espectrofotometría de emisión atómica por plasma inductivo arrojan $0.56 \mathrm{mg}$ de cobre por cada $100 \mathrm{~g}$ (Pedro et al., 2006); estudios con té verde determinaron contenido de $0.492 \mathrm{mg} / 100 \mathrm{~g}$ (Citak y Tuzen, 2010).

El cobre contenido en el extracto enzimático no puede atribuirse exclusivamente a la enzima Polifenoloxidasa. Pero la actividad de esta enzima es potenciada por la presencia del cobre en ese extracto. El cobre es el cofactor por excelencia de la PFO. Un fruto que contiene polifenoles y la enzima de PFO está destinado a presentar obscurecimiento, pero un fruto que además tiene un alto contenido de cobre anuncia un obscurecimiento acelerado (Es-Safi et al., 2003). El aguacate revela un mayor contenido de cobre en la fruta que las demás muestras, con $0.292 \mathrm{mg} \mathrm{Cu} / 100 \mathrm{~g}$ reportado en la Tabla 1 . Por el contrario la salsa de tomate, cuyo fruto también contiene PFO, tiene $0.080 \mathrm{mg} \mathrm{Cu} / 100 \mathrm{~g}$ de cobre y su deterioro no es perceptible con facilidad (Casado-Vela et al. 2005). El contenido de cobre de los frutos con PFO advierte un mayor o menor obscurecimiento dependiendo del pH del fruto.

Sin embargo, el obscurecimiento es distinto para frutas con pH ácido. Así, la ciruela, la fresa y el kiwi presentan un obscurecimiento menos perceptible que el aguacate y la pera debido al $\mathrm{pH}$ ácido, ya que éste inhibe la actividad de la PFO. El contenido de cobre en dichas frutas es bajo: 
0.119 ppm en ciruela, 0.094 ppm en fresa y 0.130 ppm en kiwi; pero la reacción entre la enzima y los polifenoles que se favorece por el cofactor de cobre también induce el desarrollo de microorganismos que llevan al deterioro y descomposición de las frutas (Casado-Vela et al. 2005).

Los resultados reportados por este estudio coinciden con otros trabajos. Investigaciones con jugos de fruta (piña, naranja, guayaba) presentan un promedio de $0.52 \pm 0.60 \mathrm{ppm}$ de cobre. Dichas muestras fueron digeridas con ácido nítrico concentrado por vía húmeda y la determinación de metales fue por absorción atómica por llama (Onianwa et al., 1999). Otro estudio de cuantificación de cobre que utilizó digestión de ácido nítrico por microondas y espectrofotómetro de emisión atómica por plasma inductivo acoplado a masas produjo un rango de 0.054 a $0.137 \mathrm{mg} \mathrm{Cu} / 100 \mathrm{~g}$ en olivas verdes y de 0.073 a 0.255 mg de Cu/ $100 \mathrm{~g}$ en olivas negras (Sahan et al., 2007).

El mamey presentó la menor cantidad de cobre en fruta con $0.038 \mathrm{mg}$ y en el extracto de enzimas con $0.013 \mathrm{mg}$. Estas cantidades no permitieron identificar una correlación con la velocidad de obscurecimiento. La cantidad de grasa en el fruto sugiere que el método de digestión para obtener enzimas no fue adecuado (Martins et al., 2001). El estudio de olivas verdes y negras mencionado anteriormente indica un método de digestión que incluye microondas; esos frutos tienen un contenido de materia grasa entre $18.85 \%$ y $49.62 \%$. Otra alternativa es la oxidación por vía seca en donde la muestra se calcina en mufla y posteriormente se recupera con ácidos, se digiere y se afora.

La Figura 1 muestra la absorbancia identificada en la banda $420 \mathrm{~nm}$ representativa de la PFO y la velocidad de reacción de obscurecimiento calculada de acuerdo a la Fórmula 1 (Fang et al., 2007). La absorbancia presenta un comportamiento lineal en los primeros $60 \mathrm{~s}$.

velocidad de reacción $(1 / \mathrm{s})=\frac{\text { Absorbancia }(\text { a } 420 \mathrm{~nm})}{60 \mathrm{~s}} \times \frac{\text { Vaforo }}{\text { Valicuota }}$

La absorbancia identificada en la banda PFO es proporcional a la concentración de la enzima de acuerdo a la Ley de Beer. El aguacate, el mamey y el mango presentan mayor actividad de la PFO para las frutas estudiadas. La velocidad de reacción del aguacate fue $0.751 \mathrm{~s}^{-1}$, muy superior al resto de las frutas y se explica por el $\mathrm{pH}$ neutro que favorece la actividad de la enzima (CasadoVela et al., 2005).

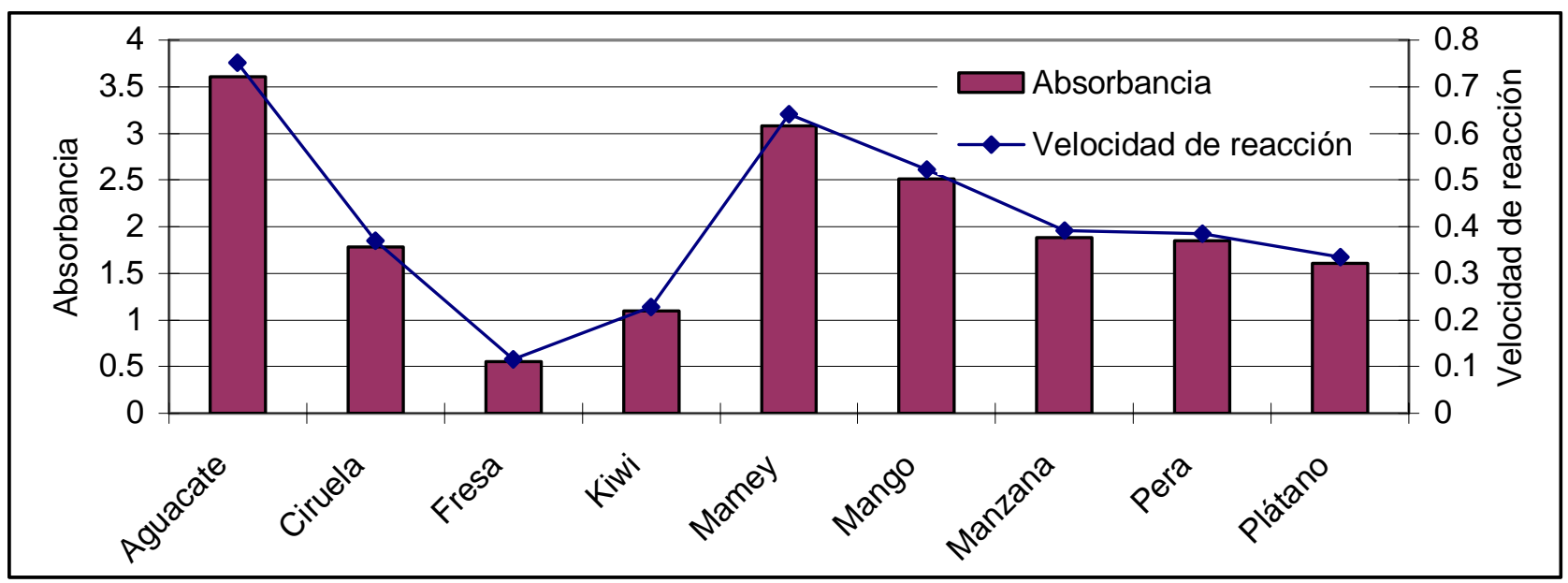

Fig. 1: Absorbancia (a $420 \mathrm{~nm}$ ) y velocidad de reacción de obscurecimiento (1/s) de frutas tropicales

Después del aguacate, el mamey obtuvo la mayor velocidad de reacción de obscurecimiento. Sin embargo, no se pudo identificar una correlación debido al menor contenido de cobre derivado del proceso inadecuado de digestión. 
En el lado opuesto de la escala de obscurecimiento, la fresa con pH de 4.03 mostró el menor contenido de cobre en fruta con $0.094 \pm 0.010 \mathrm{mg} \mathrm{Cu} / 100 \mathrm{~g}$ y en extracto con $0.016 \pm 0.010 \mathrm{mg}$ de $\mathrm{Cu} / 100 \mathrm{~g}$. El pH ácido y el bajo contenido de cobre en extracto contribuyen a la más baja tasa de reacción de obscurecimiento.

El kiwi tiene la segunda velocidad de reacción más baja, el pH más ácido en las frutas estudiadas con 3.66 y un contenido de cobre en extracto enzimático de la de mitad de tabla con $0.050 \pm 0.014$ $\mathrm{mg} \mathrm{Cu} / 100 \mathrm{~g}$. El contenido de cobre contribuye en la reacción de obscurecimiento, pero el pH tan ácido es determinante al inhibir la actividad de la PFO y el obscurecimiento de la fruta.

La manzana, con $\mathrm{pH}$ de 5.45, mostró un bajo contenido de cobre en fruta con $0.072 \mathrm{mg} \mathrm{Cu} / 100 \mathrm{~g}$ fruta y $0.031 \mathrm{mg} \mathrm{Cu} / 100 \mathrm{~g}$ en extracto. Pero su velocidad de reacción no fue tan baja como la fresa. La correlación entre el contenido de cobre y la tasa de obscurecimiento no pudo establecerse debido a la influencia del pH no tan ácido.

Tampoco pudo establecerse una correlación para el mango con el tercer lugar en la tasa de obscurecimiento a $0.522 \mathrm{~s}^{-1}$, un contenido de cobre en fruta de $0.191 \mathrm{mg} \mathrm{Cu} / 100 \mathrm{~g}$ y $0.258 \mathrm{mg}$ $\mathrm{Cu} / 100 \mathrm{~g}$ en extracto de enzimas a mitad de tabla. A pesar del pH ácido a 4.89 y el mediano contenido de cobre, la velocidad de reacción fue alta.

La pera y el plátano tienen un pH similar (5.54 y 5.60) y una velocidad media de reacción de obscurecimiento (0.385 s-1 y $0.335 \mathrm{~s}-1$ ) comparada con el resto de las frutas de estudio. Sin embargo, el contenido de cobre en extracto para la pera $(0.051 \mathrm{mg} \mathrm{Cu} / 100 \mathrm{~g})$ casi duplica el contenido de cobre en plátano $(0.029 \mathrm{mg} \mathrm{Cu} / 100 \mathrm{~g})$. Estas diferencias evidentes en la cantidad de cobre no permiten establecer una relación directa con la velocidad de reacción bajo pH similar.

Una gran cantidad de cobre en extracto enzimático tampoco es indicativo de una velocidad de reacción mayor en $\mathrm{pH}$ ácidos. El contenido de cobre en el extracto de la pera duplica al de manzanas $(0.031 \mathrm{mg} \mathrm{Cu} / 100 \mathrm{~g})$ y ciruelas $(0.033 \mathrm{mg}$ de $\mathrm{Cu} / 100 \mathrm{~g})$ pero su velocidad de reacción es menor a la presentada por la manzana $\left(0.391 \mathrm{~s}^{-1}\right)$ y mayor a la velocidad en ciruela $\left(0.370 \mathrm{~s}^{-1}\right)$. El $\mathrm{pH}$ de la manzana es 5.45 y la ciruela es 4.07 , ambos más ácidos que la pera y el plátano.

\section{CONCLUSIONES}

El contenido de cobre varía de una fruta a otra, tanto en la pulpa como en el extracto de sus enzimas. La enzima PFO causante del obscurecimiento es potenciada por el cobre: a mayor cantidad de cobre en extracto, más rápido ocurre el obscurecimiento. El aguacate y el mango muestran una relación directa entre la cantidad de cobre en extracto y la velocidad de obscurecimiento debido a su $\mathrm{pH}$ neutro.

Sin embargo, el pH ácido retardó el obscurecimiento. La ciruela, la manzana, la pera y el plátano tienen pH ácido y mostraron indicadores similares en la velocidad de reacción de obscurecimiento. Cuando las frutas tienen $\mathrm{pH}$ ácidos menores a cuatro disminuye la velocidad de oscurecimiento aunque exista suficiente cobre en forma de cofactor. El medio ácido actúa como antioxidante y retarda la reacción de oscurecimiento. El kiwi y la fresa mostraron el pH más ácido y la menor velocidad de reacción de obscurecimiento.

Dada la influencia del pH y otras condiciones (oxidación, cantidad y composición de enzimas), no se pudo establecer una correlación directa y proporcional entre el contenido natural de cobre en el extracto y la velocidad de reacción para todas las frutas en este estudio.

\section{REFERENCIAS}

AOAC International, Official methods of analysis of AOAC International, 14a edición, Association of Official Analytical Chemists International, Arlington, Estados Unidos (1984). 
Artés, F., M. Castañer y M.I. Gil, El pardeamiento enzimático en frutas y hortalizas mínimamente procesadas, Food Science and Technology Internacional: 4(6), 377-389 (1998).

Cano, P., M.A. Marin y C. Fuster, Effects of some thermal treatments on polyphenoloxidase and peroxidase activities of banana (Musa cavendishii, var enana), Journal Science Food Agriculture: 51(2), 223-231 (1990).

Casado-Vela, J., S. Sellés y R. Bru, Purification and Kinetic Characterization of Polyphenoloxidase from Tomato fruits (Lycopersicon Esculentum cv. Muchamiel), Journal of Food Biochemistry: 29, 381-401 (2005).

Citak, D. y M. Tuzen, A novel preconcentration procedure using cloud point extraction for determination of lead, cobalt and copper in water and food samples using flame atomic absorption spectrometry, Food and Chemical toxicology: 48, 1399-1404 (2010).

Es-Safi, N.E., V. Cheynier y M. Moutounet, Effect of copper on oxidation of (+)-catechin in a model solution system, Journal of food science and technology: 38, 153-163 (2003).

Fang, C., C. Wang, Y. Xiong y K.W.L. Pomper, Extraction and Characterization of Polyphenoloxidase in pawpaw (Asimina Triloba) fruit, Journal of Food Biochemistry: 31, 603-620 (2007).

Joo, S.J. y N.M. Betts, Copper intakes and consumption patterns of chocolate foods as sources of copper for individuals in the 1987-1988 nationwide food consumption survey, Nutrition Research: 16(1), 41-52 (1996).

Lamikanra, O. y M.A. Watson, Effects of ascorbic acid on peroxidase and polyphenoloxidase activities in fresh-cut cantaloupe melon, Journal of Food Science: 66(9), 1283-1286 (2001).

Martins, S.I., W.M. Jongen y M.A. Boekel, A review of Maillard reaction in food and implications to kinetic modeling, Trends in Food Science \&Technology: 11, 364-373 (2001).

Oniabwa, P. C., I.G. Adetola, C.M. Iwegbue, M.F. Ojo y O.O. Tella, Trace heavy metals composition of some Nigerian beverage and food drinks, Food Chemistry: 66, 275-279 (1999).

Pedro, N.A.R., E. de Oliveira y S. Cadore, Study of mineral content of chocolate flavored beverages, Food Chemistry: 95, 94-100 (2006).

Queiroz, Ch., M.L. Mendes, E. Fialho y V.L. Valente-Mesquita. Polyphenol Oxidasa: Characteristics and mechanisms of Browning control, Food Reviews International: 24(4), 361-375 (2008).

Sahan, Y., F. Basoglu y S. Gücer. ICP-MS análisis of a series of metales (namely: Mg, Cr, Co, Ni, $\mathrm{Fe}, \mathrm{Cu}, \mathrm{Zn}, \mathrm{Sn}, \mathrm{Cd}$ y $\mathrm{Pb}$ ) in black and green olive samples from Bursa, Turkey, Food Chemistry: 105, 395-399 (2007).

Sapp, R.E. y S.D. Davidson, Microwave digestion of multi-component foods for sodium analysis by atomic absorption spectrometry, Journal of Food Science: 25(5), 1412-1414 (1991).

Zukowska, J. y M. Biziuk. Methodological evaluation of method for dietary heavy metal intake, Journal of Food Science: 73 (2), R21-R28 (2008). 
